\title{
Nível socioeconômico e ensino superior: cálculo e aplicações
}

\author{
Erica Castilho Rodrigues \\ Daniel Abud Seabra Matos \\ Aline dos Santos Ferreira
}

Resumo: Os objetivos desta pesquisa foram: a) elaborar uma medida do nível socioeconômico para o ensino superior, a partir da técnica da Teoria de Resposta ao Item; b) ilustrar a aplicação do indicador de nível socioeconômico por meio da análise da Universidade Federal de Ouro Preto (UFOP); c) analisar e comparar o nível socioeconômico dos cursos de graduação e institutos da UFOP. Usamos os dados do Exame Nacional do Ensino Médio (ENEM) de 1.210 alunos ingressantes na UFOP em 31 cursos de graduação no primeiro semestre de 2013. Utilizamos a Teoria de Resposta ao Item, mais especificamente o modelo para respostas graduadas. Dentre os principais resultados, destacamos: a UFOP apresentou um percentual expressivo de alunos nos estratos mais baixos de nível socioeconômico; uma grande discrepância quando comparamos os cursos de graduação e institutos da UFOP; os alunos que utilizaram política afirmativa no SISU para ingressar na UFOP possuem mediana menor do nível socioeconômico em comparação com estudantes que não utilizaram política afirmativa (evidência empírica de que as cotas estão cumprindo uma função de inclusão social); a importância da utilização de indicadores educacionais como o nível socioeconômico para que a instituição possa planejar e monitorar adequadamente suas políticas de gestão educacional.

Palavras-chave: Nível socioeconômico. Teoria de resposta ao item. Ensino superior.

\section{Socioeconomic status and higher education: calculation and applications}

Abstract: The goals of this research were to: a) develop a measure of socioeconomic status for higher education, based on the technique of Item Response Theory; b) illustrate the application of the socioeconomic status indicator analyzing the Federal University of OuroPreto (UFOP); c) analyze and compare the socioeconomic status of UFOP's undergraduate courses and institutes. We used data from the National Exam of Upper Secondary Education (ENEM) of 1.210 UFOP's freshmen in 31 undergraduate courses on the first semester of 2013. We used the Item Response Theory, more specifically the graded response model. Highlight results include: UFOP presented an expressive percentage of students in the lower strata of socioeconomic status; a large discrepancy when comparing UFOP's undergraduate courses and institutes; students who used affirmative action in SISU for entry into UFOP have lower median socioeconomic status compared to students who did not use affirmative action (empirical evidence that quotas are fulfilling a social inclusion function); the importance of using educational indicators such as socioeconomic status to enable the institution to adequately plan and monitor their educational management policies.

Key words: Socioeconomic status. Item response theory. Higher education. 


\section{Introdução}

Nas últimas décadas, mudanças importantes aconteceram na educação superior brasileira, como a expansão do número de instituições (tanto privadas quanto públicas), ampliação do acesso, mudanças na legislação, no financiamento e nos processos de gestão dos sistemas de ensino. Estas mudanças também incluem aspectos relacionados à avaliação educacional. Em 2004, por exemplo, a lei 10.861 instituiu o Sistema Nacional de Avaliação da Educação Superior (SINAES) (BRASIL, 2004). No entanto, segundo Bertolin (2007), o Brasil ainda precisa avançar no que se refere a um sistema de indicadores bem estruturado para o monitoramento da qualidade da educação superior em nível de sistema, incluindo aspectos de entradas, processo e resultados do ensino superior. Como a "qualidade da educação" é um conceito múltiplo, são sempre necessários um conjunto de indicadores para avaliá-la. Neste sentido, a presente pesquisa se propõe a investigar um dos indicadores educacionais mais importantes, especialmente para a realidade brasileira: o nível socioeconômico.

O nível socioeconômico é uma variável latente, que pode ser considerada como uma síntese feita a partir da combinação de vários elementos. Variáveis latentes são aquelas que não podem ser observadas diretamente e por isso são necessários instrumentos como testes ou questionários que se associem a esta variável (SOARES, 2005). Assim, o "NSE visa mensurar empiricamente um construto teórico que situa os indivíduos em classes ou estratos sociais, nos quais eles compartilham algumas características semelhantes tais como ocupação, renda ou educação" (ALVES et al., 2013, p. 16). Entretanto, na literatura não existe um consenso sobre a definição desse construto, inclusive sobre quais dimensões precisam ser consideradas para a construção do indicador. Existem métodos diferentes de calcular o nível socioeconômico. O Critério Brasil, por exemplo, situa os indivíduos nas classes A1, A2, B1, B2,C1, C2, D ou E. O seu cálculo é simples, sendo utilizado pela Associação Brasileira de Empresas de Pesquisa (ABEP). Um exemplo internacional é o Programa Internacional de Avaliação de estudantes (PISA), que usa dados sobre a ocupação dos pais dos estudantes na construção do indicador denominado International Socio-Economic Index of Occupational Status (ISEI) (GANZEBOOM et al., 1992).

O que torna o nível socioeconômico importante na área educacional é a sua forte correlação com o desempenho dos estudantes. Desde o Relatório Coleman em 1966, pesquisas nacionais e internacionais acumularam extensas evidências da grande associação entre o nível socioeconômico das famílias e o desempenho escolar (LUZ, 2006; SIRIN, 2005; SOARES; ANDRADE, 2006; SOARES; COLLARES, 2006; WHITE, 1982). Para Silva e Hasenbalg (2002), diversas dimensões do nível socioeconômico têm um impacto direto no desempenho educacional. São dois exemplos destas dimensões:1) recursos econômicos ou capital econômico, usualmente mensurados através da renda ou riqueza familiar. A situação de bem-estar material dos domicílios, expressa pelas condições de moradia, também pode ser considerada; 2) recursos educacionais ou capital cultural, que estão relacionados à distribuição da educação entre os membros da família.

Em face da importância dessa área de estudos, realizamos uma pesquisa cujos objetivos foram: a) elaborar uma medida do nível socioeconômico para estudantes do ensino superior, a partir da técnica da Teoria de Resposta ao Item; b) ilustrar a aplicação do indicador de nível socioeconômico por meio da análise do perfil dos alunos ingressantes na Universidade 
Federal de Ouro Preto em 2013; c) analisar e comparar o nível socioeconômico dos cursos de graduação e institutos da Universidade Federal de Ouro Preto.

Vale ainda destacar que nosso trabalho foi baseado na proposta de Alves et al. (2013, 2014), que elaboraram uma metodologia de cálculo do nível socioeconômico das escolas de educação básica brasileiras. Neste sentido, a principal contribuição da nossa pesquisa é estender esta discussão para o ensino superior.

\section{Metodologia}

\subsection{Base de dados e caracterização dos sujeitos}

Os dados utilizados são oriundos dos questionários contextuais do Exame Nacional do Ensino Médio (ENEM), que coletam informações que permitem calcular o nível socioeconômico dos alunos. Mais especificamente, usamos os dados dos alunos ingressantes na Universidade Federal de Ouro Preto (UFOP) no primeiro semestre de 2013. Além disso, destacamos que apenas os alunos "computados para o censo da educação superior" foram analisados. Em outras palavras, apenas aqueles estudantes que, após as diversas chamadas de matrícula, efetivamente permaneceram na instituição.

O banco de dados foi obtido com a Pró-reitoria de Graduação (PROGRAD), sendo composto por 1.210 alunos ingressantes nos cursos de Administração, Arquitetura e Urbanismo, Artes Cênicas, Ciência da Computação, Ciência e Tecnologia de Alimentos, Ciências Econômicas, Direito, Educação Física, Engenharia Civil, Engenharia de Computação, Engenharia de Controle e Automação, Engenharia de Minas, Engenharia de Produção (campus João Monlevade e Ouro Preto), Engenharia Elétrica, Engenharia Geológica, Engenharia Mecânica, Engenharia Metalúrgica, Farmácia, Física, História, Jornalismo, Letras, Matemática, Medicina, Música, Nutrição, Pedagogia, Química Industrial, Serviço Social, Sistemas de Informação, Turismo.

Assim, calculamos o nível socioeconômico dos alunos ingressantes na UFOP em um total de 31 cursos. Destacamos que alguns cursos não aparecem nesta pesquisa porque os seus dados não estavam disponíveis no banco: Filosofia, Engenharia Ambiental, Ciências Biológicas, Estatística, Química Licenciatura, Museologia (esses cursos só tiveram entrada no segundo semestre de 2013). Já os cursos à distância - EaD (Administração Pública, Geografia, Matemática e Pedagogia) não integram o estudo porque possuem uma forma diferenciada de ingresso na instituição. No total, foram 1.333 alunos ingressantes em cursos presenciais na UFOP no primeiro semestre de 2013. A diferença em relação aos 1.210 casos analisados são alguns dados faltantes, talvez devido ao não preenchimento do questionário.

O banco original possuía 45 itens relacionados ao nível socioeconômico. Apenas um item não foi retirado do ENEM: se o aluno "usou política afirmativa" para o ingresso na instituição. Este item foi fornecido pela UFOP.

Como mencionamos anteriormente, nosso trabalho foi baseado na metodologia proposta por Alves et al. (2013, 2014). Os autores utilizaram dados de diversas avaliações educacionais (inclusive do ENEM): a Prova Brasil, o Sistema de Avaliação da Educação Básica (SAEB) e o Exame Nacional do Ensino Médio (ENEM). No entanto, apesar do nosso trabalho utilizar os dados do ENEM, a amostra que analisamos é constituída por alunos do ensino superior. Ou seja, uma amostra composta pelos estudantes que ingressaram no ensino superior pelo Sistema de Seleção Unificada (SISU). Além disso, outras diferenças da nossa pesquisa 
com relação aos trabalhos de Alves et al. $(2013,2014)$ são as aplicações e análises específicas para o ensino superior (ver seções "Análise da Universidade Federal de Ouro Preto" e "Considerações finais").

\subsection{Modelo de análise}

Para o estudo de variáveis latentes, a Teoria de Resposta ao Item (TRI) é largamente utilizada. O interesse na aplicação de técnicas derivadas da TRI vem crescendo, principalmente na área educacional. A TRI propõe modelos para os traços latentes, que são características que não podem ser observadas diretamente. Segundo Valle (2000), existem vários modelos para representar diferentes tipos de variáveis, dependendo de três fatores: 1) da natureza do item- dicotômicos ou não dicotômicos; 2) do número de populações envolvidas- apenas uma ou mais de uma; 3) da quantidade de traços latentes que está sendo medida- um ou mais de um. Neste trabalho, utilizamos o modelo para respostas graduadas, proposto por Samejima (1969), para situações em que existem mais de duas categorias ordenadas para a resposta (EMBRETSON; REISE, 2000).

De acordo com Valle (2000), as categorias de um item i são arranjados em ordem decrescente e denotados por $\mathrm{k}=0,1, \ldots, \mathrm{m}_{\mathrm{i}}$, onde $\left(\mathrm{m}_{\mathrm{i}}+1\right)$ é o número de categorias do i-ésimo item. O item i toma diferentes valores categóricos ordenados, em que a categoria $\mathrm{k}=0$ significa que o avaliado não completou nenhuma etapa e se situa na primeira categoria de resposta do item, enquanto $\mathrm{k}=\mathrm{m}_{\mathrm{i}}$ expressa que o avaliado completou todas as etapas da tarefa e se situa na última categoria de resposta do item (BAZÁN et al., 2011).

A probabilidade de um indivíduo j escolher uma categoria particular ou outra mais alta do item i pode ser dada pelo modelo logístico de dois parâmetros: $P_{i, k}^{+}\left(\theta_{j}\right)=\frac{1}{1+e^{-a_{i, k}\left(\theta_{j-} b_{i, k}\right)}}$.

$P_{i, k}^{+}\left(\theta_{j}\right)=$ É a probabilidade de um indivíduo $\mathrm{j}$ escolher a categoria $\mathrm{k}$ ou outra mais alta do item i;

$\mathrm{K}=$ Categorias de cada item; $0, \ldots, \mathrm{m}_{\mathrm{i}}$;

$\theta_{\mathrm{j}}=$ Habilidade do indivíduo j;

$a_{i}=$ é o parâmetro de discriminação comum a todas as categorias do item i

$b_{i, k}=$ é o parâmetro de dificuldade da k-ésima categoria do item i.

Por definição tem-se que $b_{i, 1} \leq b_{i, 2} \leq \cdots \leq b_{i, m_{i}}$ ou seja, as categorias mais altas terão valor maior para o parâmetro de dificuldade.

A probabilidade de um indivíduo j escolher a categoria k para o i-ésimo item é dada por: $P_{i, k}\left(\theta_{j}\right)=P_{i, k}^{+}\left(\theta_{j}\right)-P_{i, k+1}^{+}\left(\theta_{j}\right)=\frac{1}{1+e^{-a_{i, k}\left(\theta_{j-} b_{i, k}\right)}}-\frac{1}{1+e^{-a_{i, k}\left(\theta_{j-} b_{i, k+1}\right)}}$ 
Sendo que, por definição: $P_{i, 0}^{+}\left(\theta_{j}\right)=1 P_{i, m_{i}+1}^{+}\left(\theta_{j}\right)=0$.

Ou seja, a probabilidade do indivíduo escolher a primeira ou qualquer uma das categorias superiores é igual a 1 e a probabilidade dele escolher uma categoria mais elevada que a última é igual a zero. Para cada item, o número de parâmetros a ser estimado será dado pelo número de categorias de resposta.

\subsection{Análise dos itens}

Uma das maneiras de avaliar a qualidade de um item é visualizá-lo em um gráfico. Os gráficos são compostos pelas curvas que caracterizam as categorias de cada item. A curva característica do item (CCI) e a curva de informação do item são importantes ferramentas para analisar os itens e o bom ajuste do modelo utilizado. Segundo Alves et al. (2013, 2014), é importante que o modelo possua itens que tragam informação em todas as faixas de NSE. Na nossa pesquisa, as FIG. 1, 2, 3 e 4 exemplificam itens com informações diferentes sobre o nível socioeconômico.

A FIG. 1 representa a curva característica do item "possui banheiro". O eixo das ordenadas mostra a probabilidade de um indivíduo ter marcado uma das categorias dado uma determinada habilidade (NSE). O eixo das abscissas representa a habilidade (NSE) calculada para um indivíduo. Este item possui quatro categorias sendo 1 (não tenho banheiro) e 4 (3 ou mais banheiros). A FIG. 1 mostra que dado que o indivíduo tenha um nível socioeconômico muito baixo, em torno de -4 até -3 , existe alta probabilidade deste indivíduo ter marcado a categoria 1. A probabilidade de marcar esta categoria vai decrescendo com o aumento do NSE. No outro extremo, estão os indivíduos com nível socioeconômico elevado, que têm alta probabilidade de marcar a categoria 4.

A FIG. 2 é a curva de informação para o item "possui banheiro". Para Andrade et al. (2000), as curvas de informação do item permitem identificar a área da escala em que o item é mais preciso. Diferentes itens geram diferentes graus de informação sobre um traço latente (construto), sendo os itens relacionados a um menor nível da escala mais úteis para diferenciar pessoas com baixo NSE. Já itens com maior informação no maior nível da escala são mais úteis para diferenciar pessoas com alto NSE. O item "possui banheiro" traz grande informação entre indivíduos de menor nível da escala e indivíduos de maior NSE. Assim, este item é bom para discriminar pessoas de baixo nível socioeconômico e pessoas de alto nível socioeconômico. 
FIGURA 1 - Curva característica do item "possui banheiro"

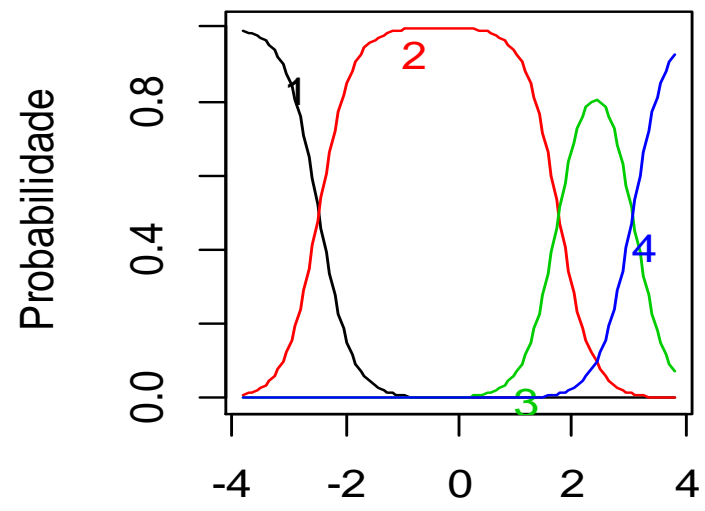

Habilidade
FIGURA 2 - Curva de informação do item "possui banheiro"

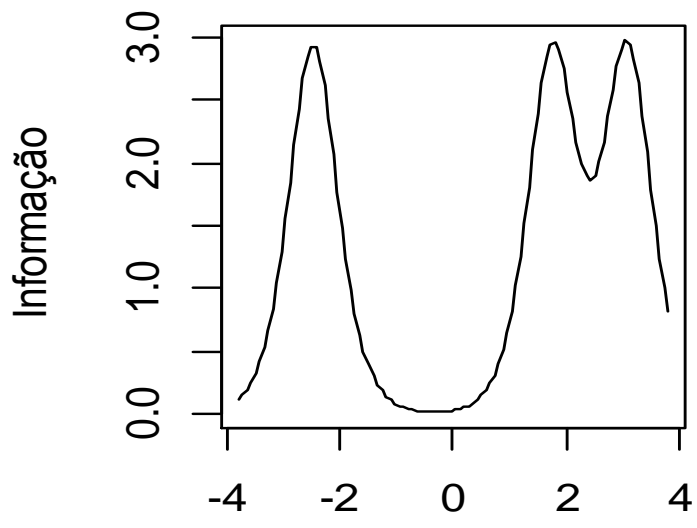

Habilidade

Fonte: elaboração dos autores.

A FIG. 3 mostra a curva característica para o item "possui TV por assinatura". Este item possui quatro categorias, sendo a categoria 1 (não tenho TV por assinatura) e a categoria 4 (Possui 3 ou mais). De acordo com esta figura, indivíduos com NSE variando de -1 a -4 tem grande probabilidade de marcar a categoria 1, sendo que de -2 a -4 a chance de marcar esta categoria é de praticamente $100 \%$. Portanto, existe pouca variação nesta faixa de NSE e consequentemente pouca informação (FIG.4). O item "possui TV por assinatura" traz grande informação entre indivíduos de NSE mediano e elevado.

FIGURA 3 - Curva característica para o item por assinatura"

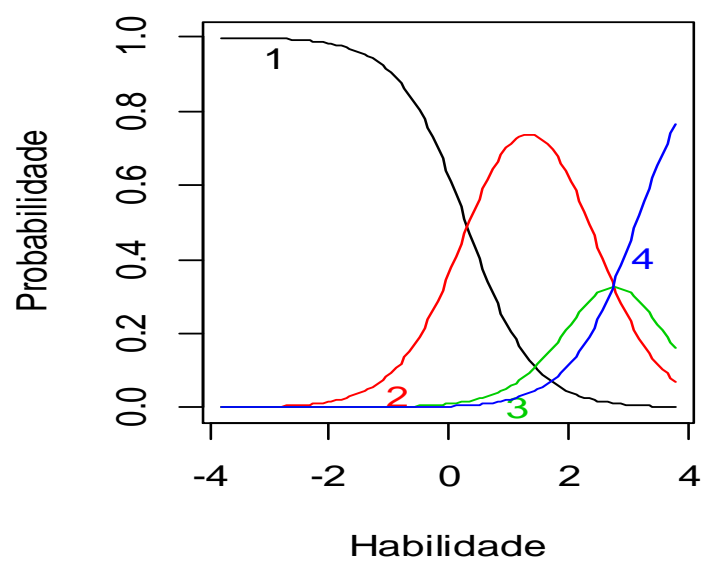

FIGURA 4 - Curva de informação para "possui TV o item "possui TV por assinatura"

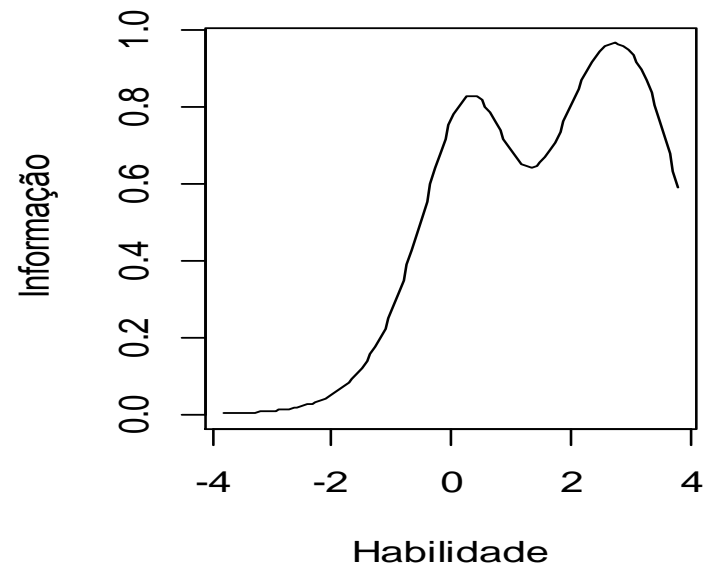

Fonte: elaboração dos autores.

Todos os itens foram analisados graficamente. Esses resultados podem ser solicitados aos autores. 


\section{Resultados}

\subsection{Seleção dos itens}

O banco de dados inicial foi composto por 45 itens. Para a aplicação do modelo de resposta gradual é necessário que as categorias de um item i estejam arranjados em ordem decrescente e denotados por $\mathrm{k}=0,1, \ldots, \mathrm{m}_{\mathrm{i}}$, onde $\left(\mathrm{m}_{\mathrm{i}}+1\right)$ é o número de categorias do i-ésimo item (VALLE, 2000). Após a ordenação das categorias dos itens, foi ajustado o modelo no software R versão 3.0.1. Para a seleção dos itens, primeiramente optamos por excluir alguns itens respondidos por uma proporção muito pequena de alunos. Além disso, após uma análise empírica, excluímos itens com discriminação negativa e itens com parâmetro de discriminação baixo (abaixo de 1). Três itens com parâmetro de discriminação muito próximos de 1 foram mantidos (TAB. 1).

Segundo Valle (2000), itens com parâmetro de discriminação negativo não são adequados, pois denotariam que um indivíduo com alta habilidade $(\theta)$ tivesse baixa probabilidade de acertar o item. Uma interpretação para o modelo de resposta gradual seria que um indivíduo com alto nível socioeconômico teria baixa probabilidade de marcar as categorias mais elevadas do NSE.

Com relação aos itens com parâmetro de discriminação considerado baixo, quanto mais próximo de 0 menos informação o item traz para o modelo (ANDRADE et al., 2000). Parâmetros de discriminação iguais a zero indicam que um candidato com baixo NSE tem a mesma probabilidade de responder a categoria mais elevada dos itens que um candidato com alto NSE. No modelo final, mantivemos 23 itens (TAB. 1).

Tabela 1 - Discriminação dos itens mantidos no modelo final

\begin{tabular}{|c|c|c|c|}
\hline Itens & $\begin{array}{l}\text { Discri- } \\
\text { minação }\end{array}$ & Itens & $\begin{array}{l}\text { Discri- } \\
\text { minação }\end{array}$ \\
\hline Você tem em sua casa? & 3,429 & Você tem em sua casa? & 1,506 \\
\hline Banheiro & & Aspirador de pó & \\
\hline Geladeira & 3,422 & Telefone fixo & 1,415 \\
\hline Empregada mensalista & 3,411 & TV em cores & 1,406 \\
\hline Acesso à internet & 2,14 & Videocassete e/ou DVD & 1,386 \\
\hline $\begin{array}{c}\text { Qual é a renda mensal de sua família? } \\
\text { (Some a sua renda com a dos seus } \\
\text { familiares) }\end{array}$ & 1,973 & $\begin{array}{c}\text { Caso você ingresse no Ensino Superior } \\
\text { privado, pretende recorrer aos auxílios } \\
\text { abaixo para custeio das mensalidades? } \\
\text { Pró-Uni }\end{array}$ & 1,273 \\
\hline $\begin{array}{l}\text { Você tem em sua casa? } \\
\text { Microcomputador }\end{array}$ & 1,915 & Até quando seu pai estudou? & 1,263 \\
\hline TV por assinatura & 1,801 & $\begin{array}{c}\text { Você tem em sua casa? } \\
\text { Telefone celular }\end{array}$ & 1,062 \\
\hline Automóvel & 1,731 & Até quando sua mãe estudou? & 1,041 \\
\hline $\begin{array}{c}\text { Em que tipo de escola você cursou o } \\
\text { Ensino Fundamental? }\end{array}$ & 1,706 & Etnia pretos/pardos & 0,974 \\
\hline $\begin{array}{c}\text { Em que tipo de escola você cursou o } \\
\text { Ensino Médio? }\end{array}$ & 1,649 & Etnia indígena & 0,974 \\
\hline $\begin{array}{l}\text { Você tem em sua casa? } \\
\text { Máquina de lavar roupa }\end{array}$ & 1,6 & Usou política afirmativa* & 0,937 \\
\hline $\begin{array}{l}\text { Freezer (aparelho independente ou parte } \\
\text { da geladeira duplex) }\end{array}$ & 1,551 & & \\
\hline
\end{tabular}

Fonte: elaboração dos autores.

Nota: * item fornecido pela Universidade Federal de Ouro Preto. 
3.2 Cálculo do nível socioeconômico e criação de grupos

As estatísticas descritivas do nível socioeconômico estão na TAB. 2. A média para estes dados é de 0,007 . O valor mínimo encontrado correspondente a um NSE mais baixo é de 3,631 e o valor máximo correspondente ao NSE mais elevado é de 3,767.

Tabela 2 - Estatísticas descritivas do NSE

\begin{tabular}{cccccccc}
\hline & $N$ & Mínimo & 1ㅇ Quartil & Mediana & Média & 3ํQuartil & Máximo \\
\hline $\begin{array}{c}\text { NSE estimado para } \\
\text { indivíduos, escala -4 a 4 }\end{array}$ & 1210 & $-3,631$ & $-0,670$ & $-0,035$ & 0,007 & 0,699 & 3,767 \\
\hline
\end{tabular}

Fonte: elaboração dos autores.

Os valores de NSE em sua escala original variam entre -4 e 4 aproximadamente. Porém, esse tipo de escala dificulta a interpretação dos resultados e gera dúvidas sobre o que significaria um valor negativo. Em vista disso, nos baseamos no trabalho de Alves et al. $(2013,2014)$ e fizemos a seguinte conversão dos dados:

$N S E 10=\left[\frac{\text { ValororiginaldoNSE-ValorminimodoNSE }}{\text { ValormáximodoNSE-ValorminimodoNSE }}\right] \times 10$.

Após esta conversão na escala de 0 a 10, geramos novas estatísticas descritivas (TAB. 3). O valor mínimo de NSE encontrado ficou próximo de 0 e o valor máximo igual a10, representando respectivamente os indivíduos com menor e maior NSE encontrados no estudo.

Tabela 3 - Estatísticas descritivas do NSE convertido na escala de 0 a 10

\begin{tabular}{|c|c|c|c|c|c|c|c|}
\hline & $\mathrm{N}$ & Mínimo & 1ㅇ Quartil & Mediana & Média & 3 Quartil & Máximo \\
\hline \multicolumn{8}{|l|}{ NSE estimado para } \\
\hline indivíduos, escala -4 a 4 & 1210 & 0.0003 & 4.002 & 4.86 & 4.918 & 5.853 & 10 \\
\hline
\end{tabular}

Fonte: elaboração dos autores.

Após o cálculo do nível socioeconômico e a conversão para uma nova escala, é de grande utilidade a divisão dos indivíduos em grupos. Uma maneira comum de separar dados em grupos distintos é utilizar os percentis, mas é necessário que a distribuição dos dados seja normal. A FIG. 5 mostra a distribuição do NSE.

Para testar se a distribuição do nível socioeconômico segue uma distribuição normal, aplicamos o teste não paramétrico de Shapiro-Wilk, resultando na rejeição da hipótese de normalidade (os dados não seguem uma distribuição normal).

Seguindo a proposta de Alves et al. $(2013,2014)$, essa divisão foi feita utilizando o método de agrupamento não hierárquico, k-médias. Este método determina a classificação dos $\mathrm{n}$ indivíduos em $\mathrm{k}$ grupos que terão homogeneidade interna e heterogeneidade externa. Com base na análise dos dados, encontramos que o número de grupos $\mathrm{k}$ deveria ser igual a 7 . A TAB. 4 mostra a frequência de indivíduos em cada um dos grupos. 
FIGURA 5 - Histograma do nível socioeconômico

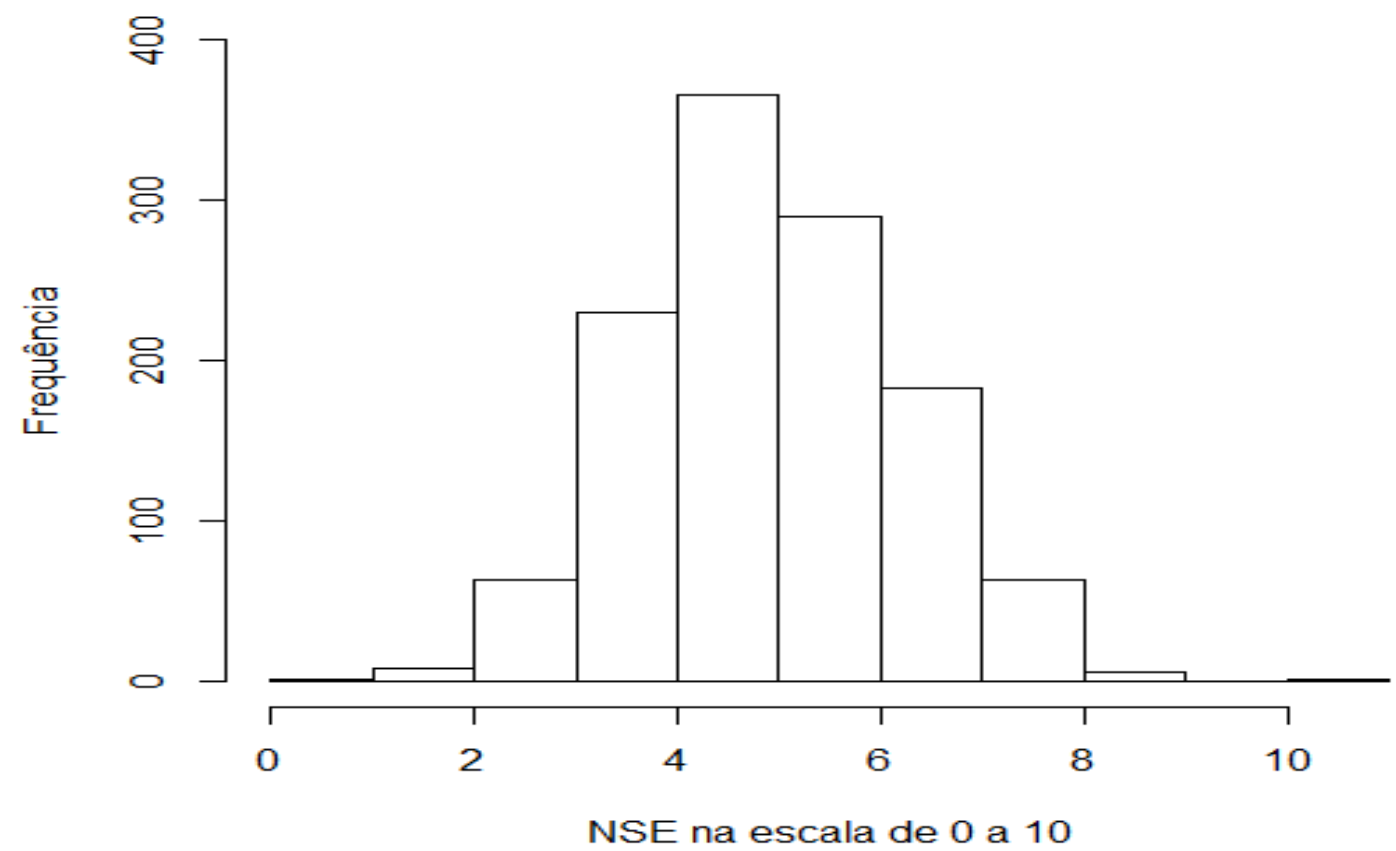

Fonte: elaboração dos autores.

TABELA 4 - Frequência de indivíduos por grupos de NSE

\begin{tabular}{cccc}
\hline Grupos & Frequência & Percentual & Percentual acumulado \\
\hline Mais baixo & 171 & 14,13 & 14,13 \\
Baixo & 237 & 19,59 & 33,72 \\
Médio baixo & 230 & 19,00 & 52,72 \\
Médio & 166 & 13,72 & 66,44 \\
Médio alto & 199 & 16,45 & 82,89 \\
Alto & 150 & 12,40 & 95,29 \\
Mais alto & 57 & 4,71 & 100,00 \\
Total & 1.210 & 100,00 & \\
\hline
\end{tabular}

Fonte: elaboração dos autores.

O grupo "baixo" tem a maior frequência de indivíduos, representando 19,59\% do total de alunos. Somados, os grupos "mais baixo", "baixo" e "médio baixo" representam 52,72\% de estudantes. Esse já é um resultado significativo da pesquisa, pois a UFOP apresentou um percentual expressivo de alunos ingressantes em 2013 nos estratos mais baixos de nível socioeconômico. Isso gera implicações inclusive nas políticas de gestão educacional da instituição. 


\subsection{Análise da Universidade Federal de Ouro Preto}

Nesta seção, apresentaremos diversas possibilidades de aplicação do indicador de nível socioeconômico no ensino superior por meio da Universidade Federal de Ouro Preto. Primeiramente, apresentamos o nível socioeconômico médio dos cursos de graduação da UFOP (TAB. 5).

Tabela 5 - Nível socioeconômico médio dos cursos de graduação da UFOP

\begin{tabular}{|c|c|c|c|}
\hline Cursos & $\begin{array}{c}\text { NSE } \\
\text { médio }\end{array}$ & Cursos & NSE médio \\
\hline Medicina & 5,72 & Nutrição & 4,88 \\
\hline Engenharia geológica & 5,64 & História (Bacharelado) & 4,82 \\
\hline Engenharia civil & 5,58 & Química industrial & 4,81 \\
\hline Engenharia de minas & 5,58 & Turismo & 4,73 \\
\hline \multirow[t]{2}{*}{ Arquitetura e urbanismo } & 5,54 & História (Licenciatura) & 4,72 \\
\hline & & Sistemas de informação & 4,68 \\
\hline Engenharia metalúrgica & 5,39 & Ciências econômicas & 4,63 \\
\hline Direito & 5,37 & Ciência e tecnologia de alimentos & 4,57 \\
\hline Engenharia de controle e automação & 5,18 & Física (Licenciatura) & 4,57 \\
\hline Física (Bacharelado) & 5,14 & Música (Licenciatura) & 4,57 \\
\hline Farmácia & 5,14 & Engenharia de computação & 4,56 \\
\hline Engenharia de produção- OP & 5,11 & Letras & 4,39 \\
\hline Engenharia elétrica & 5,08 & Matemática (Licenciatura) & 4,30 \\
\hline Ciência da computação & 5,06 & Pedagogia - licenciatura & 4,23 \\
\hline Engenharia de produção- JM & 5,03 & Serviço social & 4,17 \\
\hline Administração & 5,02 & Educação física & 4,09 \\
\hline Jornalismo & 5,02 & Artes cênicas & 3,8 \\
\hline Engenharia mecânica & 4,95 & Matemática (Bacharelado) & 2,96 \\
\hline
\end{tabular}

Fonte: elaboração dos autores.

Nota: OP= Ouro Preto. JM= João Monlevade.

Como indicado na TAB. 5, os alunos do curso de Matemática (Bacharelado) possuem o menor nível socioeconômico médio $(2,96)$. No outro extremo, estão os estudantes de Medicina, com o maior nível socioeconômico médio $(5,72)$. Sobre a TAB. 5, é necessário explicar um fator importante. Como a média é uma medida estatística muito sensível a valores extremos, a melhor forma de entender a realidade e comparar os cursos de graduação é observar a distribuição dos alunos nas 7 faixas de NSE: 1- "Mais Baixo"; 2- "Baixo"; 3-"Médio-Baixo"; 4- "Médio"; 5- "Médio-Alto"; 6- "Alto"; 7- "Mais alto" (ALVES et al., 2013, 2014). Neste sentido, apresentamos alguns exemplos na FIG. 6. 
FIGURA 6 - Distribuição dos alunos nas faixas de NSE por curso

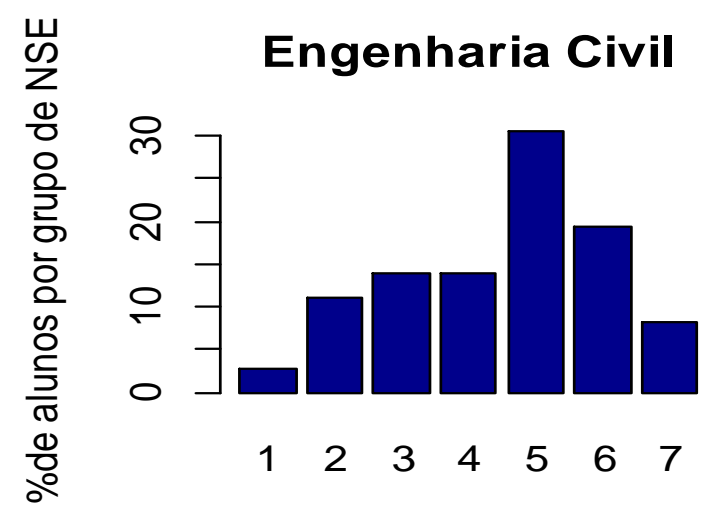

Grupos de NSE
Engenharia de Minas

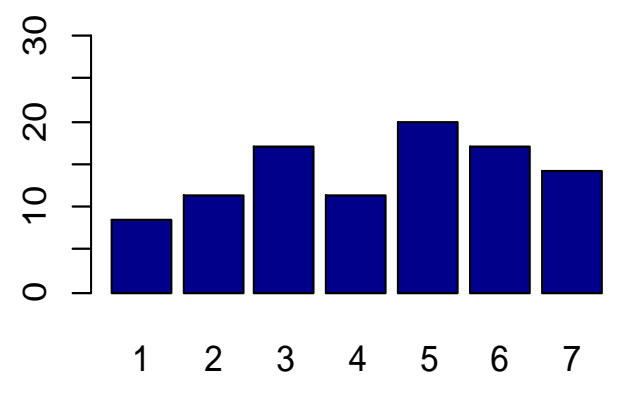

Grupos de NSE

\section{Arquitetura e Urbanismo}

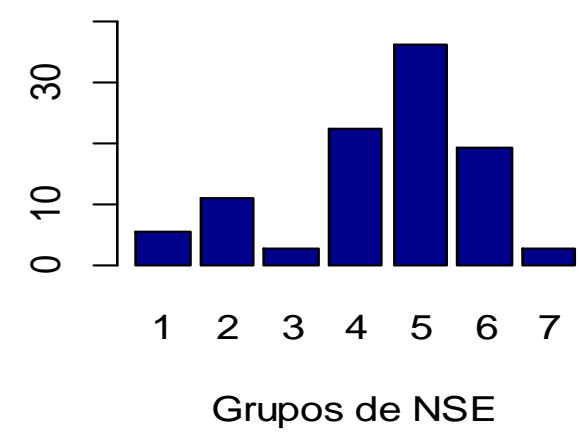

Fonte: elaboração dos autores.

Os cursos de Engenharia Civil e Engenharia de Minas possuem o mesmo nível socioeconômico médio $(5,58)$. Já o curso de Arquitetura e Urbanismo possui NSE médio praticamente idêntico aos outros dois cursos de engenharia. Entretanto, a FIG. 6 mostra que a distribuição dos alunos nas faixas de NSE são bem diferentes nos três cursos. Arquitetura e Urbanismo possui a maior concentração nos estratos mais altos do NSE, enquanto Engenharia de Minas apresenta a distribuição nas faixas de NSE mais equilibrada dentre os 3 cursos (os gráficos de todos os cursos de graduação podem ser solicitados diretamente aos autores). Dados como estes evidenciam que seria importante que o nível socioeconômico fosse incorporado nas instituições de ensino superior juntamente com outros indicadores mais utilizados como aprovação, reprovação e evasão.

Outra análise comparativa possível em instituições de ensino superior se refere às unidades acadêmicas. Atualmente, a UFOP possui 10 unidades acadêmicas, mais os cursos isolados vinculados administrativamente à Reitoria, distribuídas nas cidades de Ouro Preto, Mariana e João Monlevade. São 46 cursos de graduação presenciais e 4 cursos na modalidade à distância. O QUADRO 1 apresenta a descrição dos cursos agrupados por institutos e cidades: 
QUADRO 1 - Unidades acadêmicas da UFOP

\begin{abstract}
Ouro Preto
Instituto de Filosofia Artes e Cultura (IFAC): Artes Cênicas Licenciatura e Bacharelado, Filosofia Licenciatura e Bacharelado e Música Licenciatura e Bacharelado

Escola de Medicina: Medicina

Escola de Farmácia: Farmácia

Escola de Nutrição: Nutrição e Ciência e Tecnologia dos Alimentos

Escola de Minas: Arquitetura e Urbanismo, Engenharia Ambiental, Engenharia Civil, Engenharia de Controle e Automação, Engenharia Geológica, Engenharia Mecânica, Engenharia Metalúrgica, Engenharia de Minas e Engenharia de Produção

Instituto de Ciências Exatas e Biológicas (ICEB): Ciência da Computação, Ciências Biológicas Licenciatura e Bacharelado, Estatística, Física Licenciatura e Bacharelado, Matemática Licenciatura e Bacharelado, Química Industrial e Química Licenciatura

Escola de Direito, Turismo e Museologia (EDTM): Direito, Turismo e Museologia

Centro Desportivo de Ouro Preto (CEDUFOP): Educação Física Licenciatura e Bacharelado

Centro de Educação Aberta e a Distância (CEAD): Administração Pública, Geografia Licenciatura, Matemática licenciatura e Pedagogia.
\end{abstract}

\title{
Mariana
}

Instituto de Ciências Sociais Aplicadas (ICSA): Administração, Ciências Econômicas, Jornalismo e Serviço Social Instituto de Ciências Humanas e Sociais (ICHS): História Licenciatura e Bacharelado, Letras Licenciatura e Bacharelado e Pedagogia Licenciatura e Bacharelado

\section{João Monlevade}

Instituto de Ciências Exatas e Aplicadas (ICEA): Engenharia da Computação, Engenharia de Produção, Engenharia Elétrica e Sistemas de Informação

Nota: os cursos presenciais Filosofia, Engenharia Ambiental, Ciências Biológicas, Estatística, Química Licenciatura, Museologia; e os cursos à distância - EaD Administração Pública, Geografia, Matemática e Pedagogia não fizeram parte da presente pesquisa.

Assim, analisamos a distribuição dos alunos nas faixas de nível socioeconômico por institutos (TAB. 6).

Como apontado na TAB. 6, os institutos com maior concentração de alunos em faixas de nível socioeconômico mais elevado são a Escola de Minas e a Escola de Medicina. A Escola de Minas com concentração nas faixas "Alto" e "Médio alto" e a Escola de Medicina nas faixas "Médio alto" e "Alto", respectivamente. Destacam-se também os institutos IFAC e CEDUFOP, que apresentam a maior concentração de estudantes nas faixas "mais baixo" e "baixo".

Ainda com relação aos institutos, a FIG. 7 apresenta o boxplot do nível socioeconômico separado por institutos. Sobre o boxplot, a linha mais escura representa a mediana, o tamanho da caixa a variabilidade e os pontos fora da caixa indicam representações atípicas (extremas). A mediana é uma medida de tendência central que aponta o valor central de uma amostra de dados. Ela divide a metade superior dos dados a partir da metade inferior.

Como apontado na FIG. 7, o instituto com maior mediana foi a Escola de Medicina, que apresentou uma variabilidade pequena. Em seguida aparece a Escola de Minas, que já apresentou uma variabilidade um pouco maior. Os institutos com menores medianas foram o IFAC e CEDUFOP. Destacamos ainda o fato de que o IFAC apresentou uma distribuição muito assimétrica, no sentido de que grande parte dos alunos se concentraram em torno de valores mais baixos para o indicador. 
Tabela 6 - Distribuição dos alunos nas faixas de NSE por institutos

\begin{tabular}{|c|c|c|c|c|}
\hline \multirow[t]{2}{*}{ Institutos } & \multicolumn{3}{|c|}{ Faixas } & \multirow[b]{2}{*}{ Médio } \\
\hline & Mais baixo & Baixo & Médio baixo & \\
\hline CEDUFOP & $10(25,00 \%)$ & $16(40,00 \%)$ & $8(20,00 \%)$ & $4(10,00 \%)$ \\
\hline EDTM & $13(15,47 \%)$ & $10(11,9 \%)$ & $15(17,86 \%)$ & $12(14,29 \%)$ \\
\hline ESCOLA_FARMÁCIA & $3(6,25 \%)$ & $7(14,58 \%)$ & $10(20,83 \%)$ & $11(22,92 \%)$ \\
\hline ESCOLA_MEDICINA & $4(10,00 \%)$ & $4(10,00 \%)$ & $1(2,50 \%)$ & $2(5,00 \%)$ \\
\hline ESCOLA_MINAS & $22(7,72 \%)$ & $41(14,39 \%)$ & $37(12,98 \%)$ & $51(17,89 \%)$ \\
\hline ESCOLA_NUTRIÇÃO & $12(18,18 \%)$ & $15(22,72 \%)$ & $13(19,70 \%)$ & $5(7,58 \%)$ \\
\hline ICEA & $19(12,34 \%)$ & $34(22,08 \%)$ & $31(20,13 \%)$ & $19(12,34 \%)$ \\
\hline ICEB & $19(13,1 \%)$ & $29(20,00 \%)$ & $35(24,14 \%)$ & $22(15,17 \%)$ \\
\hline $\mathrm{ICHS}$ & $30(22,56 \%)$ & $28(21,05 \%)$ & $35(26,31 \%)$ & $16(12,03 \%)$ \\
\hline ICSA & $33(16,84 \%)$ & $46(23,47 \%)$ & $44(22,45 \%)$ & $22(11,22 \%)$ \\
\hline IFAC & $6(31,58 \%)$ & $7(36,84 \%)$ & $1(5,26 \%)$ & $2(10,53 \%)$ \\
\hline \multirow[t]{2}{*}{ Institutos } & \multicolumn{3}{|c|}{ Faixas } & \\
\hline & Médio alto & Alto & Mais alto & Total \\
\hline CEDUFOP & $1(2,50 \%)$ & $1(2,50 \%)$ & $0(0,00)$ & $40(100 \%)$ \\
\hline EDTM & $16(19,05 \%)$ & $11(13,10 \%)$ & $7(8,33 \%)$ & $84(100 \%)$ \\
\hline ESCOLA_FARMÁCIA & $8(16,67 \%)$ & $9(18,75 \%)$ & $0(0,00 \%)$ & $48(100 \%)$ \\
\hline ESCOLA_MEDICINA & $16(40,00 \%)$ & $8(20,00 \%)$ & $5(12,50 \%)$ & $40(100 \%)$ \\
\hline ESCOLA_MINAS & $56(19,65 \%)$ & $60(21,05 \%)$ & $18(6,32 \%)$ & $285(100 \%)$ \\
\hline ESCOLA_NUTRIÇÃO & $11(16,67 \%)$ & $7(10,61 \%)$ & $3(4,55 \%)$ & $66(100 \%)$ \\
\hline ICEA & $34(22,08 \%)$ & $14(9,09 \%)$ & $3(1,95 \%)$ & $154(100 \%)$ \\
\hline ICEB & $22(15,17 \%)$ & $16(11,03 \%)$ & $2(1,38 \%)$ & $145(100 \%)$ \\
\hline $\mathrm{ICHS}$ & $9(6,77 \%)$ & $10(7,52 \%)$ & $5(3,76 \%)$ & $133(100 \%)$ \\
\hline ICSA & $24(12,24 \%)$ & $14(7,14 \%)$ & $13(6,63 \%)$ & $196(100 \%)$ \\
\hline IFAC & $2(10,53 \%)$ & $0(0,00 \%)$ & $1(5,26 \%)$ & $19(100 \%)$ \\
\hline
\end{tabular}

Fonte: elaboração dos autores.

Também utilizamos o nível socioeconômico para comparar dois grupos: estudantes que utilizaram política afirmativa no Sistema de Seleção Unificada (SISU) para ingressar na UFOP e estudantes que não utilizaram (FIG. 8).

A FIG. 8 mostra que indivíduos que utilizaram política afirmativa no SISU para ingressar na UFOP possuem mediana menor do NSE em comparação com alunos que não utilizaram política afirmativa. Isso é uma evidência empírica de que as políticas de cotas estão cumprindo uma função esperada de inclusão social. Percebe-se ainda que os dois grupos apresentam variabilidade semelhante. Na mesma direção dos dados da UFOP, podemos comparar o nível socioeconômico de alunos cotistas e de ampla concorrência considerando todo o universo de estudantes brasileiros. Nesse sentido, consideramos os dados do Censo do Ensino Superior de 2013 ( $N=9.929 .289)$. Como o nível socioeconômico dos alunos não está disponível nessa base de dados, escolhemos três variáveis que o representariam de maneira indireta: acesso a bolsa permanência, acesso a apoio moradia e acesso a apoio transporte. Primeiramente, fizemos um teste qui-quadrado para testar a dependência de cada uma dessas variáveis com o fato do aluno ter utilizado ou não a política de cotas. Os testes mostra- 
ram que as três variáveis estão relacionadas com a política de cotas. Para a variável acesso a bolsa permanência, observou-se que dentre os cotistas 30,86\% tiveram acesso a esse benefício, enquanto na ampla concorrência apenas 4,81\% usufruíram dele. Já para a variável acesso a apoio moradia, a proporção de alunos cotistas que tiveram acesso ao benefício foi de $15,69 \%$, enquanto na ampla concorrência foi de $2,81 \%$. Por fim, na variável acesso a apoio de transporte, essas proporções foram de $28,28 \%$ e 14,36\%, respectivamente. Todos esses resultados evidenciam que, quando considerado o cenário brasileiro como um todo, a política de cotas parece estar atendendo estudantes com nível socioeconômico mais baixo.

\section{FIGURA 7 - Boxplot do NSE separado por institutos}

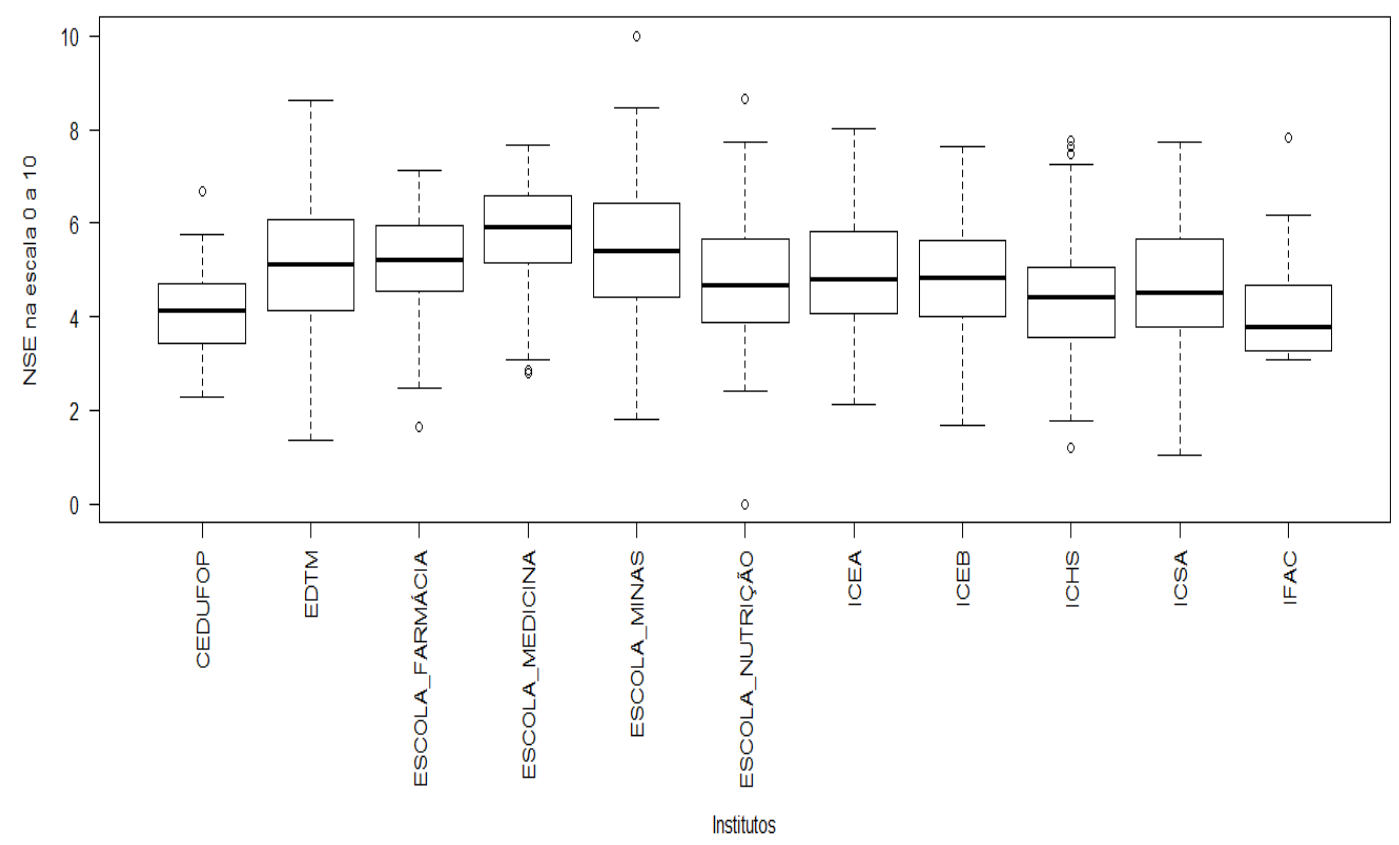

Fonte: elaboração dos autores.

FIGURA 8 -Boxplot do NSE separado por política afirmativa

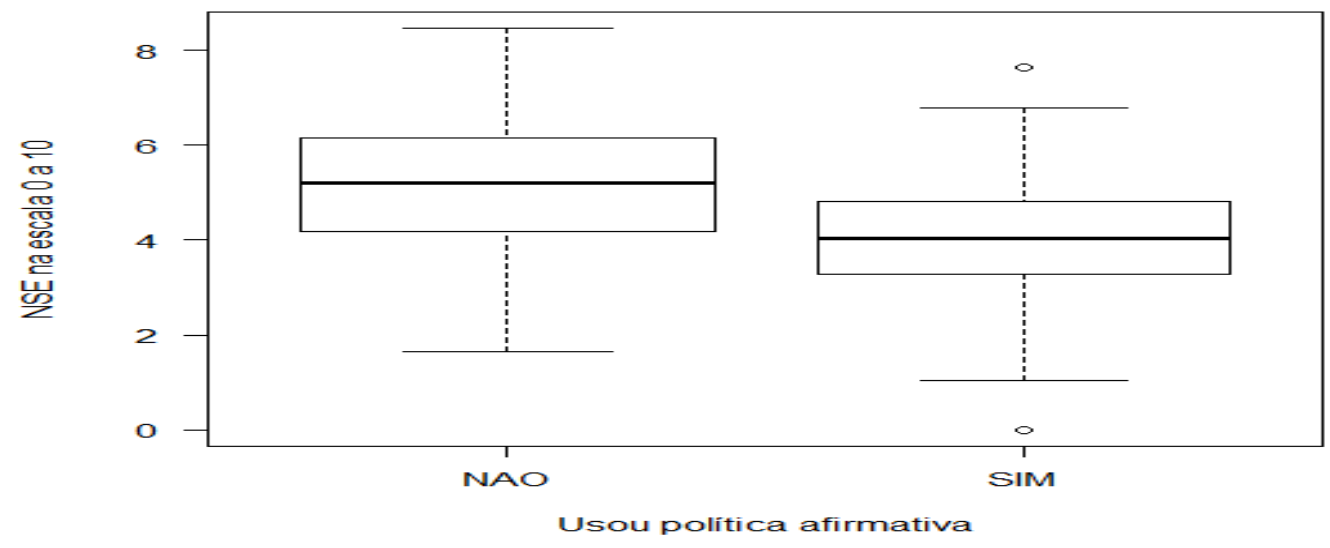

Fonte: elaboração dos autores. 
Tomados em conjunto, os resultados desta pesquisa podem impactar as políticas de gestão educacional da instituição. Toda avaliação é um processo que envolve três etapas: 1) coleta de informação; 2) análise dessa informação e conclusão sobre o resultado; 3 ) tomada de decisões de acordo com a conclusão (JORBA; SANMARTÍ, 2003). Neste sentido, é crucial que a instituição de ensino superior faça um diagnóstico preciso de seu público para poder implementar ações adequadas. Cursos e institutos diferentes podem requerer intervenções diversificadas, que respondam aos perfis dos alunos atendidos por eles. Dessa forma, cursos ou institutos com percentual elevado de estudantes de baixo nível socioeconômico podem receber maior auxílio da instituição, como, por exemplo, um número maior de bolsas. Além disso, acompanhar alunos cotistas e de ampla concorrência no decorrer dos anos pode servir tanto para monitorar o impacto da lei de cotas quanto para auxiliar estudantes que apresentem baixo desempenho na Universidade (monitorar demandas para assistência estudantil e outras políticas). Esse acompanhamento da trajetória dos alunos poderia ser facilitado por meio da incorporação do indicador de nível socioeconômico na rotina da instituição. Assim, seria interessante associar o nível socioeconômico com outros indicadores mais comuns como notas, reprovação e evasão.

\section{Considerações finais}

Neste trabalho, nos baseamos na proposta de Alves et al. (2013, 2014), que elaboraram uma metodologia de cálculo do nível socioeconômico das escolas de educação básica brasileiras. Assim, a principal contribuição da nossa pesquisa foi estender esta discussão para o ensino superior. Neste sentido, seria interessante que os mesmos procedimentos usados para o ensino básico acontecessem no ensino superior. Por exemplo: no caso do ENEM, o INEP contextualiza as medidas de aprendizado apresentando informações sobre o nível socioeconômico dos alunos das escolas de ensino médio (INEP, 2014). No caso do ensino superior, o INEP já apresenta os seguintes relatórios referentes ao Exame Nacional de Desempenho dos Estudantes (ENADE): relatórios do curso, da instituição e da área avaliada (INEP, 2015). Esses relatórios apontam diversas informações, inclusive dados sobre o nível socioeconômico. No entanto, o que os relatórios do ENADE indicam é a porcentagem de respostas das perguntas relacionadas ao NSE. Dessa forma, eles não apresentam o NSE médio do curso/instituição ou a distribuição dos alunos por faixas de NSE. Vale ainda destacar que também é possível pensar a construção do indicador de nível socioeconômico usando o questionário do ENADE. No entanto, esse seria um indicador mais limitado, pois o questionário do ENEM possui um número maior de perguntas associadas ao construto do nível socioeconômico. Nesse sentido, mesmo para o ensino superior, sugerimos o uso do indicador a partir do ENEM, já que ele representa o nível socioeconômico do aluno na sua entrada e a mobilidade social não se altera muito em um curto período como estudante. Outra desvantagem do ENADE é que ele não atinge todos os alunos todos os anos. Somente alguns cursos participam em cada ano.

Assim, produzimos uma medida do nível socioeconômico para o ensino superior, a partir da técnica da Teoria de Resposta ao Item, utilizando dados da Universidade Federal de Ouro Preto. Analisando os alunos da UFOP como um todo, a faixa de nível socioeconômico "baixo" tem a maior frequência de estudantes. Somados, os grupos "mais baixo", "baixo" e 
"médio baixo" representam 52,72\% de alunos. Ou seja: a UFOP apresentou um percentual expressivo de alunos ingressantes em 2013 nos estratos mais baixos de nível socioeconômico.

Os resultados mostraram uma grande discrepância quando comparamos os cursos e institutos da UFOP. Os alunos do curso de Artes Cênicas apresentaram o menor nível socioeconômico médio. No outro extremo, os estudantes de Medicina possuem o maior nível socioeconômico médio. Os Institutos com maior concentração de estudantes em faixas de nível socioeconômico mais elevado são a Escola de Minas e a Escola de Medicina. Já o Instituto de Filosofia Artes e Cultura (IFAC) e o Centro Desportivo de Ouro Preto (CEDUFOP) apresentam a maior concentração de estudantes nas faixas de NSE "mais baixo" e "baixo".

Quanto às políticas de cotas, os alunos que utilizaram política afirmativa no SISU para ingressar na UFOP possuem mediana menor do NSE em comparação com indivíduos que não utilizaram política afirmativa. Isso é uma evidência empírica de que as cotas estão cumprindo uma função de inclusão social.

Tomados em conjunto, os resultados desta pesquisa evidenciam a importância da utilização de indicadores educacionais como o nível socioeconômico para que a instituição possa planejar e monitorar adequadamente suas políticas de gestão educacional.

Com relação às limitações da pesquisa, como usamos os dados dos alunos ingressantes na UFOP, esses resultados não podem ser generalizados para outras instituições de ensino superior. Futuramente são necessárias mais pesquisas envolvendo um número maior de instituições de ensino superior e também a utilização de bancos de dados diversos. Outras limitações já mencionadas por Alves et al. $(2013,2014)$ também se aplicam, como o fato de que os dados para a construção de indicadores educacionais são obtidos geralmente com questionários respondidos pelos próprios estudantes.

\section{Referências}

ALVES, Maria Teresa Gonzaga; SOARES, José Francisco; XAVIER, Flavia Pereira. Índice Socioeconômico das Escolas de Educação Básica Brasileiras. Ensaio: Avaliação e Políticas Públicas em Educação, Rio de Janeiro, v. 22, n. 84, p. 671-704, 2014.

ALVES, Maria Teresa Gonzaga; SOARES, José Francisco; XAVIER, Flavia Pereira. O Nível Socioeconômico das Escolas de Educação Básica Brasileiras. In: REUNIÃO DA ASSOCIAÇÃO BRASILEIRA DE AVALIAÇÃO EDUCACIONAL - ABAVE, 7., 2013, Brasília. Anais... - Avaliação e Currículo: um diálogo necessário, 2013. p. 15-32.

ANDRADE, Dalton Francisco de; TAVARES, Heliton Ribeiro; DA CUNHA VALLE, Raquel. Teoria da resposta ao item: conceitos e aplicações. ABE, Sao Paulo, 2000.

BAZÁN, Jorge Luis; MAZZON, Jose Afonso; MERINO, Martin Hernani. Classificação de modelos de resposta ao item policotômicos com aplicação ao marketing. Revista Brasileira de Estatística, Rio de Janeiro, v. 72, n. 235, p. 7-40, 2011.

BERTOLIN, Júlio CG. Indicadores em nível de sistema para avaliar o desenvolvimento e a qualidade da educação superior brasileira. Avaliação, Campinas; Sorocaba, p. 309-331, 2007. 
BRASIL. CONGRESSO NACIONAL. Lei n. 10.861, de 14 de abril de 2004. Institui o Sistema Nacional de Avaliação da Educação Superior - SINAES e dá outras Providências. Brasília, DF. 2004 Disponível em: <http://www.inep.gov.br/superior/avaliacao_institucional/ legislacao.htm>. Acesso em: 7 fev. 2015.

COLEMAN, James S. et al. Equality of educational opportunity. Washington, DC, 1966. EMBRETSON, Susan E.; REISE, Steven P. Item response theory. Mahwah, New Jersey: Psychology Press, 2013.

GANZEBOOM, Harry B. G.; DE GRAAF, Paul M.; TREIMAN, Donald J. A standard international socio-economic index of occupational status. Social Science Research, Amsterdam, v. 21, n. 1, p. 1-56, 1992.

INSTITUTO NACIONAL DE ESTUDOS E PESQUISAS EDUCACIONAIS ANÍSIO TEIXEIRA - INEP. Indicador de Nível Socioeconômico (Inse) das escolas do Enem 2013. Brasília-DF: Inep, 2014. Nota Técnica.

INSTITUTO NACIONAL DE ESTUDOS E PESQUISAS EDUCACIONAIS ANÍSIO TEIXEIRA - INEP. Relatórios Enade. Disponível em: <

http://portal.inep.gov.br/enade/relatorios>. Acesso em: 17 fev. 2015.

JORBA, Jaume; SANMARTI, Neus. A função pedagógica da avaliação. Avaliação como apoio à aprendizagem. Porto Alegre: Artmed, 2003.

LUZ, Luciana Soares. Os determinantes do desempenho escolar: a estratificação educacional e o efeito valor adicionado. In: ENCONTRO NACIONAL DE ESTUDOS POPULACIONAIS, Caxambú, v. 15, 2006.

SAMEJIMA, Fumiko. Estimation of latent ability using a response pattern of graded scores. Richmond, VA, 1969. (Psychometric Monograph No. 17). Psychometric Society. Retrieved from: < http://www.psychometrika.org/journal/online/MN17.pdf>

SILVA, Nelson do Valle; HASENBALG, Carlos. Recursos familiares e transições educacionais. Cad. Saúde Pública, Rio de Janeiro, v. 18, p. 67-76, 2002.

SIRIN, Selcuk R. Socioeconomic status and academic achievement: a meta-analytic review of research. Review of Educational Research, Los Angeles, v. 75, n. 3, p. 417-453, 2005.

SOARES, Jose Francisco; ANDRADE, Renato Júdice de. Nível socioeconômico, qualidade e eqüidade das escolas de Belo Horizonte. Ensaio: avaliação e políticas públicas em educação, Rio de Janeiro, v. 14, n. 50, p. 107-126, 2006.

SOARES, José Francisco; COLLARES, Ana Cristina Murta. Recursos familiares e o desempenho cognitivo dos alunos do ensino básico brasileiro. Dados, Rio de Janeiro, v. 49, n. 3, p. 1-23, 2006.

SOARES, Tufi Machado. Utilização da teoria da resposta ao item na produção de indicadores sócio-econômicos. Pesquisa Operacional, Rio de Janeiro, v. 25, n. 1, p. 83-112, 2005.

VALLE, Raquel da Cunha; Teoria da resposta ao item. Estudos em Avaliação Educacional, São Paulo, v. 21, p. 7-91, 2000. 
WHITE, Karl R. The relation between socioeconomic status and academic achievement. Psychological bulletin, Washington, DC, v. 91, n. 3, p. 461, 1982.

Erica Castilho Rodrigues - Universidade Federal de Ouro Preto Ouro Preto |MG | Brasil. Contato: ericarodrigues@iceb.ufop.br

Daniel Abud Seabra Matos - Universidade Federal de Ouro Preto Mariana |MG | Brasil. Contato: danielmatos@ichs.ufop.br

Aline dos Santos Ferreira - Universidade Federal de Ouro Preto Ouro Preto | MG | Brasil. Contato: alinee.sferreiraa@gmail.com aprovado em 13 de fevereiro de 2017. 